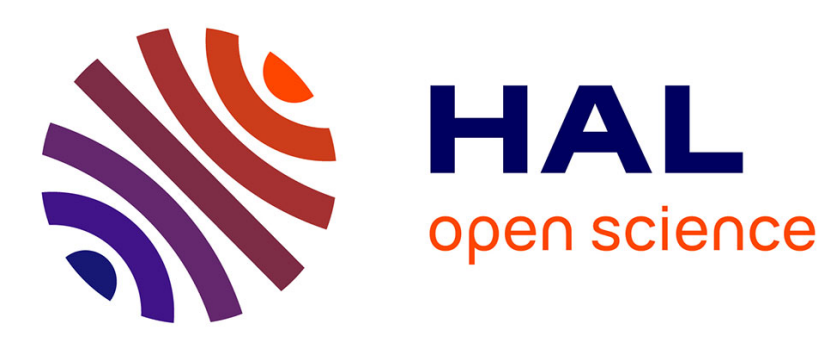

\title{
On the topology defined by Thurston's asymmetric metric
}

Athanase Papadopoulos, Guillaume Théret

\section{To cite this version:}

Athanase Papadopoulos, Guillaume Théret. On the topology defined by Thurston's asymmetric metric. Mathematical Proceedings of the Cambridge Philosophical Society, 2007, 142 (3), pp.487-496. 10.1017/S0305004107000023 . hal-00129728

\section{HAL Id: hal-00129728 \\ https://hal.science/hal-00129728}

Submitted on 8 Feb 2007

HAL is a multi-disciplinary open access archive for the deposit and dissemination of scientific research documents, whether they are published or not. The documents may come from teaching and research institutions in France or abroad, or from public or private research centers.
L'archive ouverte pluridisciplinaire HAL, est destinée au dépôt et à la diffusion de documents scientifiques de niveau recherche, publiés ou non, émanant des établissements d'enseignement et de recherche français ou étrangers, des laboratoires publics ou privés. 


\title{
ON THE TOPOLOGY DEFINED BY THURSTON'S ASYMMETRIC METRIC
}

\author{
ATHANASE PAPADOPOULOS AND GUILLAUME THÉRET
}

\begin{abstract}
In this paper, we establish some properties of Thurston's asymmetric metric $L$ on the Teichmüller space $\mathcal{T}_{\mathfrak{g}, \mathfrak{n}}$ of a surface of genus $\mathfrak{g}$ with $\mathfrak{n}$ punctures and with negative Euler characteristic. We study convergence of sequences of elements in $\mathcal{T}_{\mathfrak{g}, \mathfrak{n}}$ in the sense of $L$, as well as sequences that tend to infinity in $\mathcal{T}_{\mathfrak{g}, \mathfrak{n}}$. We show that the topology that the asymmetric metric $L$ induces on Teichmüller space is the same as the usual topology. Furthermore, we show that $L$ satisfies the axioms of a (not necessarily symmetric) metric in the sense of Busemann and conclude that $L$ is complete in the sense of Busemann.
\end{abstract}

AMS Mathematics Subject Classification: 30F60 ; 32G15.

Keywords: Teichmüller space, Thurston's asymmetric metric, geodesic lamination.

Consider a set $X$ equipped with a function $\delta: X \times X \rightarrow[0, \infty)$ satisfying the following three axioms:

A1: $\delta(x, x)=0$ for every $x$ in $X$;

A2: $\delta(x, y)>0$ for every $x$ and $y$ in $X$ with $y \neq x$;

A3: $\delta(x, z) \leq \delta(x, y)+\delta(y, z)$ for every $x, y$ and $z$ in $X$.

Of course, such a function $\delta$ is a metric in the usual sense if it is symmetric, that is, if it satisfies the following additional axiom:

A4: $\delta(x, y)=\delta(y, x)$ for every $x, y$ in $X$.

We shall call an asymmetric metric a function $\delta: X \times X \rightarrow[0, \infty)$ that satisfies A1, A2 and A3 and that does not satisfy A4, that is, such that there exist $x$ and $y$ in $X$ such that $\delta(x, y) \neq \delta(y, x)$. We note that in the theory of Finsler spaces, such a function is usually called an irreversible metric.

It is easy to make general definitions, but a definition is useful only if one can find interesting examples of objects that satisfy its axioms and that do not satisfy the axioms of the more restrictive definition. A beautiful example of an asymmetric metric is Thurston's asymmetric metric on Teichmüller space, introduced in [8]. In this paper, we shall study the topology on Teichmüller space induced by this asymmetric metric.

We shall recall below the definition of that metric, and before that we fix a few notations.

Date: July 6, 2006. 
Let $\mathfrak{g}$ and $\mathfrak{n}$ be two nonnegative integers and let $S=S_{\mathfrak{g}, \mathfrak{n}}$ be a connected oriented surface of negative Euler characteristic, obtained from a closed surface of genus $\mathfrak{g}$ by removing $\mathfrak{n}$ points which we shall call the punctures. A hyperbolic metric on $S$ is a Riemannian metric of constant curvature -1. The Teichmüller space of $S$ is the space of equivalence classes of complete finite area hyperbolic metrics on $S$, where two metrics are considered equivalent if they are isotopic, that is, if one of these metrics is the image of the other one by a map which is isotopic to the identity. The Teichmüller space of $S$ is denoted by $\mathcal{T}(S)=\mathcal{T}_{\mathfrak{g}, \mathfrak{n}}$. This space carries a natural topology that makes it homeomorphic to $\mathbb{R}^{6 \mathfrak{g}-6+2 \mathfrak{n}}$. In what follows, we shall refer to this topology as the usual topology of Teichmüller space. We briefly recall one way of defining it, by explicitly describing a homemorphism $\mathcal{T} \rightarrow$ $\mathbb{R}^{6 \mathfrak{g}-6+2 \mathfrak{n}}$. We start by taking a set of closed geodesics decomposing $S$ into hyperbolic pairs of pants. Here, a hyperbolic pair of pants is a sphere with three open disks of dimension zero or two removed, such that each boundary component is either a closed geodesic or a cusp, the cusps being at the punctures of $S$. The decomposition provides a set of parameters for the space $\mathcal{T}(S)$, where two parameters are associated to each geodesic, one parameter $($ in $(0, \infty))$ being the length of that geodesic, and the other parameter (in $\mathbb{R}$ ) being a twist parameter. A twist parameter is defined up to the choice of an origin corresponding to the zero-twist. This collection of parameters gives a homeomorphism between $\mathcal{T}(S)$ and $\mathbb{R}^{3 \mathfrak{g}-3+\mathfrak{n}} \times(0, \infty)^{3 \mathfrak{g}-3+\mathfrak{n}}$. For a detailed description of this homeomorphism in the case $\mathfrak{n}=0$, we refer the reader to [9] p. 271 . One can also refer to [1], [3] and [4]. These length-twist parameters associated to pair of pants decompositions are usually called FenchelNielsen parameters.

We now recall the definition of Thurston's asymmetric metric.

For any two hyperbolic metrics $g$ and $h$ on $S$ and for any diffeomorphism $\varphi: S \rightarrow S$ which is isotopic to the identity, we consider the Lipschitz constant $\operatorname{Lip}(\varphi)$ of $\varphi$, defined by

$$
\operatorname{Lip}(\varphi)=\sup _{x \neq y \in S} \frac{d_{h}(\varphi(x), \varphi(y))}{d_{g}(x, y)} .
$$

The infimum of the Lipschitz constants associated to all diffeomorphisms $\varphi$ that are isotopic to the identity is denoted by

$$
L(g, h)=\log \inf _{\varphi \sim \operatorname{Id}_{S}} \operatorname{Lip}(\varphi) .
$$

Making $g$ and $h$ vary in their respective homotopy classes, we obtain a function (which we denote by the same letter)

$$
L: \mathcal{T}(S) \times \mathcal{T}(S) \rightarrow[0, \infty)
$$

which is Thurston's asymmetric metric. 
It is easy to see that the function $L$ satisfies the triangle inequality, but it requires some work to see that $L(g, h)>0$ for any $g \neq h$ (see [8], Theorem 3.1).

There is another geometrically defined measure of a distance between two metrics $g$ and $h$, which was introduced by Thurston, which we shall denote by $K(g, h)$. We now recall its definition.

A closed curve in $S$ is called essential if it is not homotopic to a point or to a puncture of $S$. We let $\mathcal{S}$ be the set of isotopy classes of simple essential closed curves in $S$.

For any homotopy class $\alpha$ of essential simple closed curves on $S$, we consider the quantity

$$
r_{g, h}(\alpha)=\frac{l_{h}(\alpha)}{l_{g}(\alpha)}
$$

where $l_{h}(\alpha)$ denotes the length of the closed geodesic in the homotopy class $\alpha$ with respect to the hyperbolic metric $h$, and we set

$$
K(g, h)=\log \sup _{\alpha \in \mathcal{S}} r_{g, h}(\alpha) .
$$

Here also, the function $K$ which is defined on the set of pairs of hyperbolic structures gives a well-defined function (which we denote by the same letter $K$ ) defined on pairs of elements of Teichmüller space.

We let $\mathcal{M} \mathcal{L}_{0}(S)$ be the space of compactly supported measured geodesic laminations on $S$, equipped with its usual topology, and we let $\mathcal{P \mathcal { L }} \mathcal{L}_{0}(S)$ be the corresponding projective space, that is, the quotient of $\mathcal{M} \mathcal{L}_{0}(S)$ by the natural action of the positive reals. We refer the reader to [7] for the definitions and the basic notions concerning these spaces. We shall also use notions like lengths and intersection functions between measured foliations, closed geodesics and compactly supported geodesic laminations, which are now classical notions, and we refer the reader to references such as [7], [8], [6] and [3], for the details.

Using the extension of the length of a closed curve to the length of a measured geodesic lamination, we have the following expression of $K(g, h)$

$$
K(g, h)=\log \sup _{\alpha \in \mathcal{M} \mathcal{L}_{0}(S)} r_{g, h}(\alpha)=\log \sup _{\alpha \in \mathcal{P \mathcal { M } \mathcal { L } _ { 0 } ( S )}} r_{g, h}(\alpha) .
$$

The last expression for $K$ is often more useful than Expression 2 because, by compactness of the space $\mathcal{P} \mathcal{M} \mathcal{L}_{0}(S)$, the supremum in 3 is attained.

It is easy to see that $K \leq L$. In his paper [8], Thurston proved that $K=L$. He also showed that the function $L$ is not symmetric in general, by considering the structures $g$ and $h$ shown in Figure 1. Figure 2 gives an example of distinct hyperbolic metrics $g$ and $h$ satisfying $K(g, h)=K(h, g)$. 
We also need to recall the following notions from Thurston's paper [8].

For any simple closed geodesic $\gamma$ in $S$ endowed with a hyperbolic metric, we can construct (in a non-canonical way) a geodesic lamination $\mu$ which satisfies the following three properties:

(1) the lamination $\mu$ has no other transverse measure of compact support than the Dirac transverse measure supported on $\gamma$;

(2) each leaf of $\mu \backslash \gamma$ is bi-infinite and each of its ends either spirals around $\gamma$ or converges to a cusp of $S$;

(3) the lamination $\mu$ is complete.

The last condition means that each connected component of the complement of $\mu$ in $S$ is isometric to the interior of a hyperbolic ideal triangle. (We recall that a hyperbolic ideal triangle is the convex hull of three distinct points in the boundary at infinity of hyperbolic plane $\mathbb{H}^{2}$.)

The lamination $\mu$ is called a completion of $\gamma$ and the closed geodesic $\gamma$ is called the stump of $\mu$. An example of a completion of a simple closed geodesic $\gamma$ is given in Figure 3 .

Finally, we recall the construction of the horocyclic measured foliation that is associated to a complete geodesic lamination. We start with a construction in a hyperbolic ideal triangle. Any such triangle is equipped with a canonical partial measured foliation which is called its horocyclic foliation. The term partial means here that the support of

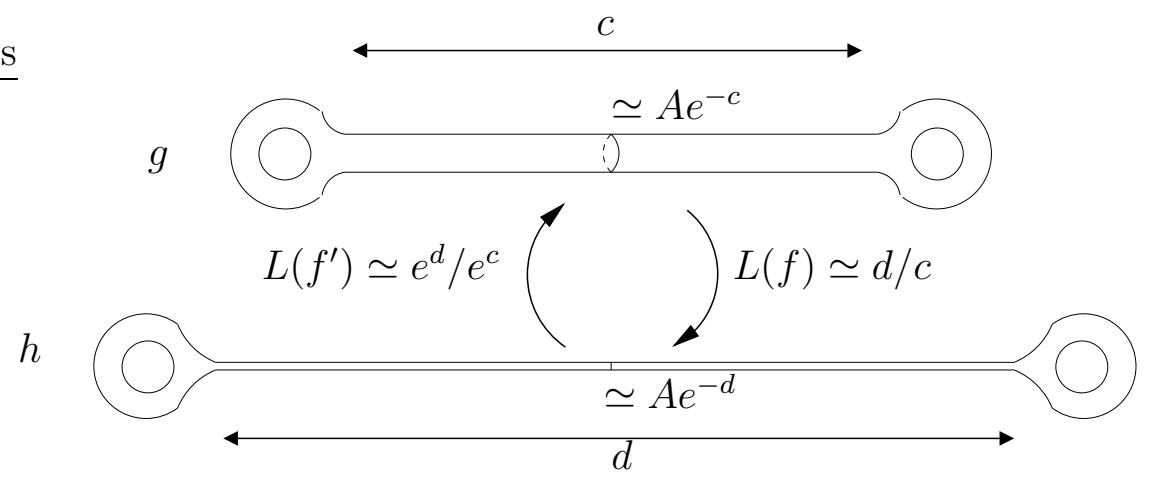

Figure 1. An example (due to Thurston) of hyperbolic sutructures $g$ and $h$ satisfying $L(g, h) \neq L(h, g)$.
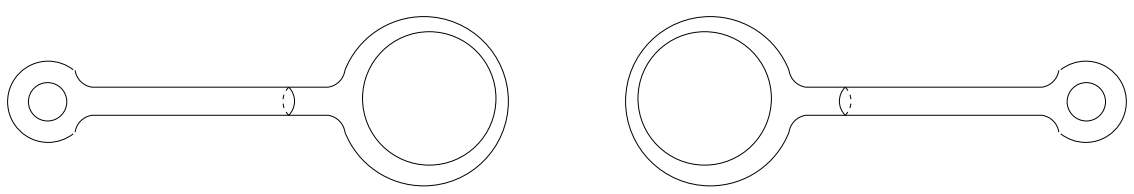

FiguRE 2. An example of distinct hyperbolic structures $g$ and $h$ satisfying $K(g, h)=K(h, g)$. Here, $h$ is the image of $g$ by an order-two isometry which is not isotopic to the identity. 
the foliation is a subset of the ideal triangle. The horocyclic foliation is characterized by the following three properties:

(1) the leaves are pieces of horocycles that are perpendicular to the edges;

(2) the non-foliated region is a triangle bordered by three of these pieces of horocycles (see Figure 4);

(3) the transverse measure assigned to any arc which is contained in an edge coincides with the Lebesgue measure induced from the hyperbolic metric.

Now if $\mu$ is any complete geodesic lamination on the hyperbolic surface $S$, then, the metric completion of each connected component of $S \backslash \mu$ being an ideal triangle, we can equip it with its canonical horocyclic measured foliation. The collection of the horocyclic measured foliations associated to these various ideal triangles fit together compatibly and define a partial measured foliation of $S$, which we call the horocyclic measured foliation associated to $\mu$. Collapsing each nonfoliated triangular region onto a tripod, we obtain a genuine (i.e. not partial) measured foliation on the surface, which is well-defined up to isotopy and which we denote by $F_{\mu}(S)$.

The fact that the hyperbolic structures that we consider on $S$ are complete and of finite area is equivalent to the fact that the horocyclic foliation $F_{\mu}(S)$ associated to $\mu$ is trivial around the punctures, that is, each puncture of $S$ has a neighborhood homeomorphic to an annulus on which the foliation induced by $F_{\mu}(S)$ consists of closed leaves

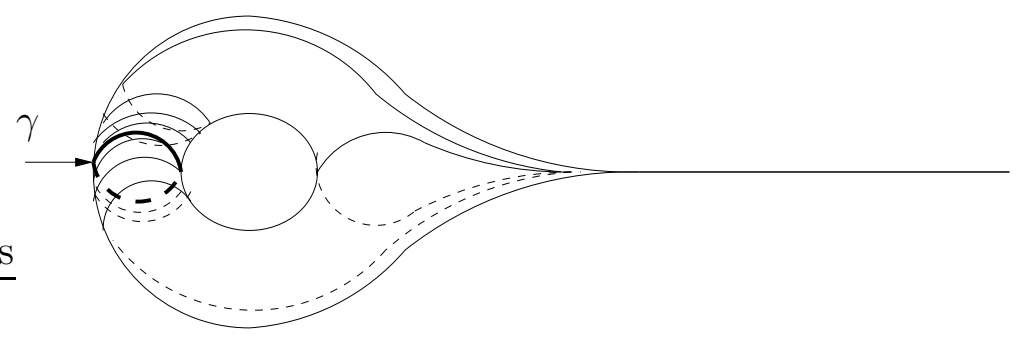

FiguRE 3. A complete lamination whose stump is a simple closed geodesic $\gamma$.

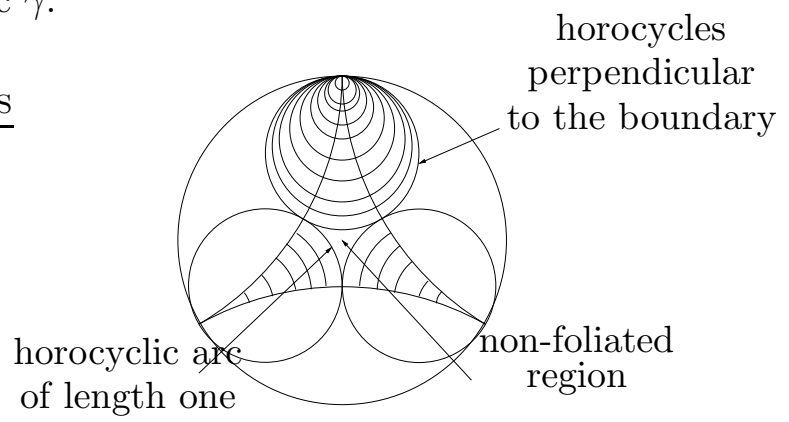

FIGURE 4. The horocyclic foliation of an ideal triangle. 
that are parallel to the puncture. Using the canonical map between measured foliations on $S$ that are trivial around the punctures and (possibly empty) compactly supported measured geodesic laminations on $S$, we obtain a well-defined compactly supported measured geodesic lamination associated to the measured foliation $F_{\mu}(S)$. We shall call it the horocyclic measured geodesic lamination associated to $\mu$, and we shall denote it by $\lambda_{\mu}(S)$.

In what follows, we establish some properties of Thurston's asymmetric metric $L$ with respect to sequences in $\mathcal{T}(S)$ that tend to infinity, and we discuss the topology that this asymmetric metric induces on that space. Here, as usual, we say that a sequence $\left(g_{n}\right)$ in $\mathcal{T}(S)$ tends to infinity (and we write $g_{n} \rightarrow \infty$ ) if for any compact subset $\mathcal{K}$ of $\mathcal{T}(S)$, we have $g_{n} \in \mathcal{T}(S) \backslash \mathcal{K}$ for all $n$ large enough.

We shall use the following lemma (see [3] p. 147).

Lemma 1. For every sequence $\left(g_{n}\right)$ of elements in $\mathcal{T}(S)$ that tends to infinity, we can find a finite set $\left\{\gamma_{1}, \ldots, \gamma_{k}\right\} \subset \mathcal{S}$ such that $\sum_{j=1}^{k} i\left(g_{n}, \gamma_{j}\right) \rightarrow$ $\infty$ as $n \rightarrow \infty$ (or, equivalently, $\left.\lim \sup _{j=1}^{k} i\left(g_{n}, \gamma_{j}\right)=\infty\right)$.

We prove the following

Theorem 1. For any sequence $\left(g_{n}\right)$ in $\mathcal{T}(S)$, the following three properties are equivalent:

(1) $g_{n} \rightarrow \infty$;

(2) for all $h \in \mathcal{T}(S), L\left(h, g_{n}\right) \rightarrow \infty$;

(3) for all $h \in \mathcal{T}(S), L\left(g_{n}, h\right) \rightarrow \infty$.

Proof. Let us first note that from the triangle inequality (which is satisfied by the asymmetric metric), we can replace in Properties 2 and 3 the statement "for all $h \in \mathcal{T}(S)$ " by "there exists $h \in \mathcal{T}(S)$ ".

We start by proving $1 \Rightarrow 2$. If $g_{n} \rightarrow \infty$, then, by Lemma 1 , for each $n \geq 0$, we can find an element $\gamma_{n}$ which we can choose among a finite set of elements in $\mathcal{S}$ such that $l_{g_{n}}\left(\gamma_{n}\right) \rightarrow \infty$ as $n \rightarrow \infty$. Since the homotopy classes $\gamma_{n}$ are chosen among finitely many elements, for any fixed $h$ in $\mathcal{T}(S)$, the value $l_{h}\left(\gamma_{n}\right)$ is bounded from above. Therefore, we have

$$
L\left(h, g_{n}\right)=\log \sup _{\lambda \in \mathcal{M} \mathcal{L}_{0}(S)} \frac{l_{g_{n}}(\lambda)}{l_{h}(\lambda)} \geq \log \frac{l_{g_{n}}\left(\gamma_{n}\right)}{l_{h}\left(\gamma_{n}\right)} \rightarrow \infty .
$$

Now let us prove $2 \Rightarrow 1$. Equivalently, we prove that if there exists $h \in \mathcal{T}(S)$ satisfying $L\left(h, g_{n}\right) \rightarrow \infty$, then $g_{n} \rightarrow \infty$. We reason by contradiction. Assume that $g_{n} \nrightarrow \infty$. This means that there exists a subsequence of $\left(g_{n}\right)$, which we also denote by $\left(g_{n}\right)$, and a compact subset $\mathcal{K}$ of $\mathcal{T}(S)$ such that $g_{n} \in \mathcal{K}$ for all large enough $n$. By compactness of $\mathcal{K}$ and, again, up to taking a subsequence of $\left(g_{n}\right)$, we can assume that this sequence converges to some point $g$ in $\mathcal{K}$. By compactness 
of $\mathcal{P} \mathcal{L}_{0}(S)$, for every $n \geq 0$, we can find an element $\eta_{n} \in \mathcal{M} \mathcal{L}_{0}(S)$ satisfying $\sup _{\lambda \in \mathcal{M} \mathcal{L}_{0}(S)} \frac{l_{g_{n}}(\lambda)}{l_{h}(\lambda)}=\frac{l_{g_{n}}\left(\eta_{n}\right)}{l_{h}\left(\eta_{n}\right)}$.

Again by compactness of $\mathcal{P} \mathcal{L}_{0}(S)$ and up to taking a subsequence of $\left(g_{n}\right)$, we can assume that the sequence of projective classes of $\eta_{n}$ converges to the projective class of some element $\gamma \in \mathcal{M} \mathcal{L}_{0}(S)$. This implies

$$
L\left(h, g_{n}\right)=\lim _{n \rightarrow \infty} \sup _{\lambda \in \mathcal{M} \mathcal{L}_{0}(S)} \frac{l_{g_{n}}(\lambda)}{l_{h}(\lambda)}=\frac{l_{g}(\gamma)}{l_{h}(\gamma)}<\infty,
$$

which is a contradiction.

The proof of $3 \Rightarrow 1$ is analogous to the proof of $2 \Rightarrow 1$.

So far, we have proved $1 \Longleftrightarrow 2 \Longleftarrow 3$. In order to prove $1 \Longrightarrow 3$, we need the following

Lemma 2. If $\left(g_{n}\right)$ is a sequence in $\mathcal{T}(S)$ tending to infinity, then there exists a sequence $\left(\lambda_{n}\right)_{n \geq 0} \in \mathcal{M} \mathcal{L}_{0}(S)$ such that

(1) the sequence $\left(l_{g_{n}}\left(\lambda_{n}\right)\right)$ is bounded from above;

(2) for all $h \in \mathcal{T}(S), l_{h}\left(\lambda_{n}\right) \rightarrow \infty$ as $n \rightarrow \infty$.

Proof. Let $\left(g_{n}\right)_{n \geq 0}$ be a sequence in $\mathcal{T}(S)$ that tends to infinity. By Lemma 1, there exists a finite set $\Gamma$ of homotopy classes of simple closed curves such that for each $n \geq 0$, there exists $\gamma_{n} \in \Gamma$ such that $l_{g_{n}}\left(\gamma_{n}\right) \rightarrow \infty$

For each $n \geq 0$, we complete $\gamma_{n}$ into a complete geodesic lamination $\mu_{n}$ of stump $\gamma_{n}$ and we let $\lambda_{n}=\lambda_{\mu_{n}}\left(g_{n}\right)$.

We know that $l_{g_{n}}\left(\lambda_{n}\right)$ is bounded. Indeed, by Lemma 3.9 and Proposition 3.3 of [5], this quantity is bounded by $-6 \chi(S)$, where $\chi(S)$ is the Euler characteristic of $S$. (The proof is given there for closed surfaces, but the arguments work more generally for surfaces of finite type.)

Let $h \in \mathcal{T}(S)$. We show that there exists $\epsilon(h)>0$ such that $l_{h}\left(\lambda_{n}\right) \geq$ $\epsilon(h) l_{g_{n}}\left(\gamma_{n}\right)$.

Let us fix an integer $n \geq 0$. We can cover the surface $S$ with a finite union of rectangles $R_{1}, \ldots, R_{N(n)}$ of disjoint interiors, such that $\lambda_{n}$ induces on each rectangle a lamination by geodesic segments whose endpoints are on the vertical sides of that rectangle and such that $\gamma_{n}$ crosses each such rectangle from one horizontal side to the other horizontal side (see Figure 5).

We can assume that the number $N(n)$ of rectangles is bounded from above, by a constant that only depends on the topology of $S$. (We can use a train track to build the rectangular cover, and in that case the number $N(n)$ is bounded by the maximal number of edges of a train track on $S$.)

For each $j \in\{1, \ldots, N(n)\}$, let $k_{n}^{j}=\sharp\left\{\gamma_{n} \cap R_{j}\right\}$ and let $w_{n}^{j}$ be the "width" of the rectangle $R_{j}$, that is, the total measure of any of its vertical sides with respect to the transverse measure $d \lambda_{n}$ of $\lambda_{n}$.

We have: 
(1) $l_{h}\left(\lambda_{n}\right)=\sum_{j=1}^{N(n)} \int_{\partial R_{j} \cap \lambda_{n}} l_{h}(x) d \lambda_{n}$, where $\left\{\partial R_{j}\right\}_{j=1, \ldots, N(n)}$ denotes the set of vertical edges of the rectangles glued together and where $l_{h}(x)$ denotes the $h$-length of the segment contained in the leaf of $\lambda_{n}$ which crosses $\partial R_{j}$ at the point $x$;

(2) $l_{g_{n}}\left(\gamma_{n}\right)=\sum_{j=1}^{N(n)} k_{n}^{j} w_{n}^{j}$

Statement 1 above is clear from the definitions. We now prove 2. For this, it is useful, for each $n \geq 0$, to replace the measured lamination $\lambda_{n}$ by a measured foliation $F_{n}$ having the same properties with respect to the rectangles $R_{1}, \ldots, R_{N(n)}$, that is, inducing on each such rectangle a foliation by segments that join its vertical sides, such that the total $F_{n}$-mass of the vertical side of each rectangle is equal to its total $\lambda_{n^{-}}$ mass. The closed geodesic $\gamma_{n}$ is in minimal position with respect to $F_{n}$, that is, there is no closed disk in $S$ whose boundary is the union of a segment in a leaf of $F_{n}$ and a segment in $\gamma_{n}$. This follows from the fact that $\gamma_{n}$, being a geodesic, was already in minimal position with respect to $\lambda_{n}$. Thus, $i\left(F_{n}, \gamma_{n}\right)$ is equal to the total $F_{n}$-mass of $\gamma_{n}$. Therefore, we have

$$
\begin{aligned}
\sum_{j=1}^{N(n)} k_{n}^{j} w_{n}^{j} & =i\left(F_{n}, \gamma_{n}\right) \\
& =i\left(F_{\mu_{n}}\left(g_{n}\right), \gamma_{n}\right)
\end{aligned}
$$

Now since $\gamma_{n}$ is the stump of $\mu_{n}$, the last quantity is equal to $l_{g_{n}}\left(\gamma_{n}\right)$. (This is Lemma 3.12 of [5] adapted to the case where the lamination

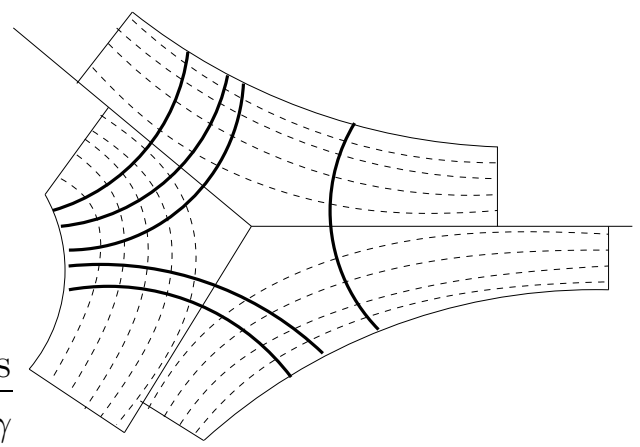

FiguRE 5. A rectangular cover of the surface, used in the proof of Lemma 2. The leaves of $\lambda_{n}$ are represented in dotted lines and the intersection of the rectangles with $\gamma_{n}$ is represented in bold lines. The width of a rectangle $R$ is the transverse measure of the vertical edges of $R$. 
$\mu_{n}$ does not necessarily have a transverse measure of full support, with $\gamma_{n}$ being its stump.) This completes the proof of 2 .

The hyperbolic structure $h$ being fixed, any geodesic segment in $\lambda_{n}$ that starts and ends at points on $\gamma_{n}$ has its length bounded below by a positive number $\epsilon(h)>0$ that is independent of $n$. This follows from the compactness of $\gamma_{n}$ and the fact that $\gamma_{n}$ is chosen among a finite set of homotopy classes of simple closed curves. Hence,

$$
\begin{aligned}
l_{h}\left(\lambda_{n}\right) & \geq \epsilon(h) \sum_{j=1}^{N(n)} k_{n}^{j} \int_{\partial R_{j} \cap \lambda_{n}} d \lambda_{n} \\
& \geq \epsilon(h) \sum_{j=1}^{N(n)} k_{n}^{j} w_{n}^{j}=\epsilon(h) l_{g_{n}}\left(\gamma_{n}\right) .
\end{aligned}
$$

Therefore, $\lim _{n \rightarrow \infty} l_{h}\left(\lambda_{n}\right)=\infty$.

Now we can complete the proof of Theorem 1.

If $g_{n} \rightarrow \infty$, then we have

$$
L\left(g_{n}, h\right)=\log \sup _{\lambda \in \mathcal{M} \mathcal{L}_{0}(S)} \frac{l_{h}(\lambda)}{l_{g_{n}}(\lambda)} \geq \log \frac{l_{h}\left(\lambda_{n}\right)}{l_{g_{n}}\left(\lambda_{n}\right)} \geq \log \frac{l_{h}\left(\lambda_{n}\right)}{C} \rightarrow \infty,
$$

as $n \rightarrow \infty$. (Here, $C$ denotes a constant bounding $l_{g_{n}}\left(\lambda_{n}\right)$ from above.)

Theorem 2. For any sequence $\left(g_{n}\right)$ in $\mathcal{T}(S)$ and for any element $g$ in $\mathcal{T}(S)$, the following three properties are equivalent:

(1) $g_{n} \rightarrow g$;

(2) $L\left(g, g_{n}\right) \rightarrow 0$;

(3) $L\left(g_{n}, g\right) \rightarrow 0$.

Proof. We start with the implication $1 \Longrightarrow 2$.

If $g_{n} \rightarrow g$, then for all $\alpha \in \mathcal{S}, r_{g, g_{n}}(\alpha)=\frac{l_{g_{n}}(\alpha)}{l_{g}(\alpha)} \rightarrow 1$. This implies that $L\left(g, g_{n}\right) \rightarrow 0$. Indeed, let us fix $\varepsilon>0$. There exists $\alpha \in \mathcal{S}$ such that $L\left(g, g_{n}\right)-\log r_{g, g_{n}}(\alpha) \leq \varepsilon$. Now there exists $N(\alpha)$ such that, for all $n \geq N(\alpha),\left|\log r_{g, g_{n}}(\alpha)\right| \leq \varepsilon$. Therefore, for all $\varepsilon>0$, there exists $N$ such that, for all $n \geq N, L\left(g, g_{n}\right) \leq 2 \varepsilon$. This proves the claim.

The proof of $1 \Longrightarrow 3$ is dealt with exactly as for $1 \Longrightarrow 2$.

So far, we proved the following implications

$$
L\left(g, g_{n}\right) \rightarrow 0 \Longleftarrow g_{n} \rightarrow g \Longrightarrow L\left(g_{n}, g\right) \rightarrow 0 .
$$

Before completing the proof of Theorem 2, we prove a result on the compactness of closed balls for Thurston's asymmetric metric.

Let $g$ be a point in $\mathcal{T}(S)$ and let $R$ be a positive real. Since $L$ is asymmetric, there are two different notions of closed balls, called the left closed ball centered at $g \in \mathcal{T}(S)$ of radius $R$ and the right closed 
ball centered at $g \in \mathcal{T}(S)$ of radius $R$, which we denote respectively by ${ }_{g} B(R)$ and $B_{g}(R)$. They are defined by

$$
{ }_{g} B(R)=\{h \in \mathcal{T}(S) \mid L(g, h) \leq R\}
$$

and

$$
B_{g}(R)=\{h \in \mathcal{T}(S) \mid L(h, g) \leq R\} .
$$

We show that these balls are compact, for the usual topology on Teichmüller space.

Proposition 3. For any $g$ in $\mathcal{T}(S)$ and for any $R>0$, the closed balls ${ }_{g} B(R)$ and $B_{g}(R)$, centered at $g \in \mathcal{T}(S)$ and of radius $R$, are compact for the usual topology.

Proof. The proof is based on Theorem 1, and arguments concerning ${ }_{g} B(R)$ and $B_{g}(R)$ are identical. We therefore only prove the compactness of ${ }_{g} B(R)$.

First note that ${ }_{g} B(R)$ is closed for the usual topology. Indeed, let $\left(g_{n}\right)$ be a sequence in ${ }_{g} B(R)$ converging to $h \in \mathcal{T}(S)$ for the usual topology. Then $L(g, h) \leq L\left(g, g_{n}\right)+L\left(g_{n}, h\right) \leq R+L\left(g_{n}, h\right)$. The implication $g_{n} \rightarrow h \Longrightarrow L\left(g_{n}, h\right) \rightarrow 0$ gives $L(g, h) \leq R$, that is, $h \in_{g} B(R)$. Therefore, ${ }_{g} B(R)$ is closed for the usual topology.

Let $\left(g_{n}\right)$ be a sequence of ${ }_{g} B(R)$. By Theorem $1, g_{n} \nrightarrow \infty$. Therefore, there exists an infinite subsequence of $\left(g_{n}\right)$, which is contained in a compact subset $\mathcal{K}$ of $\mathcal{T}(S)$ for the usual topology. We also call $\left(g_{n}\right)$ this subsequence. Up to taking another subsequence, we may assume that $g_{n} \rightarrow h$. Since $g_{n} \in{ }_{g} B(R)$ and since ${ }_{g} B(R)$ is closed, $h \in{ }_{g} B(R)$, which proves that ${ }_{g} B(R)$ is a compact subset of $\mathcal{T}(S)$.

We now complete the proof of Theorem 2 .

We must prove that $L\left(g, g_{n}\right) \rightarrow 0 \Longrightarrow g_{n} \rightarrow g$.

If $L\left(g, g_{n}\right) \rightarrow 0$, then there exists $R>0$ such that, for all $n \in \mathbb{N}$, $g_{n} \in{ }_{g} B(R)$. Since ${ }_{g} B(R)$ is compact, the sequence $\left(g_{n}\right)$ has at least one cluster point $h \in{ }_{g} B(R)$ (for the usual topology). Let $\left(g_{n}\right)$ also denote a subsequence converging to $h$. We have $L(g, h) \leq L\left(g, g_{n}\right)+L\left(g_{n}, h\right)$. Passing to the limit as $n$ goes to infinity, we obtain $L(g, h)=0$. By Theorem 3.1 of [8], $g=h$. This implies that $g$ is the cluster point of $\left(g_{n}\right)$. Therefore, $g_{n} \rightarrow g$.

The proof of $L\left(g_{n}, g\right) \rightarrow 0 \Longrightarrow g_{n} \rightarrow g$ can be done in a similar way. This completes the proof of Theorem 2 .

Consider the symmetrization $\sigma L: \mathcal{T}(S) \times \mathcal{T}(S) \rightarrow[0, \infty)$ of $L$, defined by the formula:

$$
\sigma L(g, h)=\max \{L(g, h), L(h, g)\}
$$


Thus, $\sigma L$ is a genuine metric on Teichmüller space, and the following is an immediate consequence of Theorem 2 .

Corollary 4. For any sequence $\left(g_{n}\right)$ in Teichmüller space and for any $h$ in that space, we have $L\left(g_{n}, h\right) \rightarrow 0$ if and only if $L\left(h, g_{n}\right) \rightarrow 0$ if and only if $\sigma L\left(h, g_{n}\right) \rightarrow 0$ if and only if $g_{n}$ converges to $h$ in the usual topology of Teichmüller space.

Since we have an asymmetric metric on Teichmüller space, it is natural to consider two topologies on that space, the first one being the topology generated by the collection of right open balls, and the second one being the topology generated by the collection of left open balls in that space. Here, a left (respectively right) open ball is defined as in 4 (respectively 5) above, except that the weak inequality is replaced by a strict inequality. For any asymmetric metric $\delta: X \times X: \rightarrow[0, \infty)$, we denote by $\delta^{*}: X \times X: \rightarrow[0, \infty)$ the asymmetric metric that is dual to $\delta$, that is, the asymmetric metric defined by $\delta^{*}(x, y)=\delta(y, x)$ for every $x$ and $y$ in $X$. We consider the topology on Teichmüller space that is generated by the collection of right open balls as being the one that is associated to Thurston's asymmetric metric $L$. Then, the topology generated by the collection of left open balls is the one associated to the dual asymmetric $L^{*}$. It is easy to see, from Axioms A1, A2 and A3 satisfied by a nonsymmetric metric, that the following (which is well-known for a genuine metric) holds: a sequence $\left(g_{n}\right)$ of points in Teichmüller space converges to a point $g$ in that space for the topology generated by the left (respectively right) open balls if and only if we have $L\left(g, g_{n}\right) \rightarrow 0$ (respectively $L\left(g_{n}, g\right) \rightarrow 0$ ) as $n \rightarrow \infty$. Therefore, we can state Corollary 4 in the following equivalent form

Corollary 5. The topologies on Teichmüller space defined by Thurston's asymmetric metric and by its dual asymmetric metric concide with the usual topology of that space.

Now from Proposition 3 and Corollaries 4 and 5, we obtain the following

Proposition 6. Teichmüller space equipped with Thurston's asymmetric metric is proper. More precisely, left and right closed balls are compact for the topology defined by that asymmetric metric.

H. Busemann developed a theory of spaces $(X, \delta)$ satisfying axioms A1, A2 and A3 and the following axiom

A4: $\delta\left(x, x_{n}\right) \rightarrow 0 \Longleftrightarrow \delta\left(x_{n}, x\right) \rightarrow 0$ for any $x$ and for any sequence $\left(x_{n}\right)$ in $X$.

On such a space, there is a natural associated topology, which is the one associated to the genuine metric $\max \{\delta(x, y), \delta(y, x)\}$. Theorem 2 guarantees that Thurston's asymmetric metric satisfies A4. We state this as follows: 
Corollary 7. Thurston's asymmetric metric satisfies Busemann's axioms.

Busemann introduced a notion of completeness for such spaces (see [2] Chapter 1). The definition is as follows. The space $(X, \delta)$ is complete if and only if for every sequence $\left(x_{n}\right)$ in $X$ satisfying $\delta\left(x_{n}, x_{n+m}\right) \rightarrow 0$ as $n$ and $m \rightarrow \infty$, the sequence converges to a point in $X$.

In that setting, the following generalization of the classical theorem of Hopf and Rinow holds (see [2] Theorem 8 p. 4):

If a locally compact space $(X, \delta)$ satisfies Axioms A1, A2 A3 and A4 and if this generalized metric $\delta$ is "intrinsic", then the following two properties are equivalent:

(1) left closed balls in $X$ are compact;

(2) $X$ is complete.

Here, the word intrinsic means that for any $x$ and $y$ in $X$, the value $\delta(x, y)$ is equal to the infimum of the lengths of all curves joining $x$ and $y$ in $X$. In this setting of generalized metrics, the length of a curve is defined in a way similar to the case of a genuine metric space. We do not need to enter into the details here, because by a result of Thurston (see [8] §6), Thurston' s asymmetric metric $L$ is geodesic (that is, any two points can be joined by an $L$-geodesic), which implies that it is intrinsic (see [2] p. 3). We should also note that a geodesic is defined here as the isometric image of an interval, except that we have to be careful about the order of the variables. More precisely, a map $\gamma:[a, b] \rightarrow \mathcal{T}(S)$, (where $[a, b]$ is a closed interval of $\mathbb{R}$ ) is a geodesic joining the two points $\gamma(a)$ and $\gamma(b)$ in $\mathcal{T}(S)$ if for all $t$ and $t^{\prime}$ in $[a, b]$ we have $\left|t-t^{\prime}\right|=L\left(\gamma(t), \gamma\left(t^{\prime}\right)\right)$.

Thus, we can state the following consequence of Proposition 6 .

Corollary 8. Thurston's asymmetric metric is complete in the sense of Busemann. 
ON THE TOPOLOGY DEFINED BY THURSTON'S ASYMMETRIC METRIC 13

\section{REFERENCES}

[1] W. Abikoff, The Real Analytic Theory of Teichmüller space, Lecture Notes in Math. 820, Springer-Verlag, 1980.

[2] H. Busemann, Recent synthetic differential geometry, Ergebnisse der Mathematik und ihrer Grenzgebiete, 54, Springer-Verlag, 1970.

[3] A. Fathi, F. Laudenbach \& V. Poénaru, Travaux de Thurston sur les surfaces, Astérisque 66-67, 1979

[4] Y. Imayoshi \& M. Taniguchi, An Introduction to Teichmüller Spaces, SpringerVerlag, Tokyo, 1992.

[5] A. Papadopoulos, On Thurston's boundary of Teichmüller space and the extension of earthquakes, Topology Appl. 41 (1991), no. 3, 147-177.

[6] R.C. Penner \& J. Harer, Combinatorics of Train Tracks, Annals of Math. Studies 125, Princeton University Press, 1992.

[7] W. P. Thurston, The geometry and topology of Three-manifolds, Mimeographed notes, Princeton University, 1976.

[8] W. P. Thurston, Minimal Stretch maps between hyperbolic surfaces, preprint (1986).

[9] W. P. Thurston, Three-dimensional geometry and topology, Vol. 1, edited by Silvio Levy, Princeton Mathematical Series 35, Princeton University Press, Princeton, NJ, 1997.

A. Papadopoulos, Institut de Recherche Mathématique Avancée, Université Louis Pasteur and CNRS, 7 rue René Descartes, 67084 StrasBourg Cedex, France

E-mail address: papadopoulos@math.u-strasbg.fr

G. Théret, Institut de Recherche Mathématique Avancée, Université Louis Pasteur and CNRS, 7 rue René Descartes, 67084 Strasbourg Cedex, France

E-mail address: theret@math.u-strasbg.fr 